\title{
ON THE GEOGRAPHY OF GORENSTEIN MINIMAL 3-FOLDS OF GENERAL TYPE*
}

\author{
MENG CHEN $^{\dagger}$ AND CHRISTOPHER D. HACON ${ }^{\ddagger}$
}

\begin{abstract}
Let $X$ be a minimal projective Gorenstein 3-fold of general type. We give two applications of an inequality between $\chi\left(\omega_{X}\right)$ and $p_{g}(X)$ :

1) Assume that the canonical map $\Phi_{\left|K_{X}\right|}$ is of fiber type. Let $F$ be a smooth model of a generic irreducible component in the general fiber of $\Phi_{\left|K_{X}\right|}$. Then the birational invariants of $F$ are bounded from above.

2) If $X$ is nonsingular, then $c_{1}^{3} \leq \frac{1}{27} c_{1} c_{2}+\frac{10}{3}$.
\end{abstract}

Key words. Canonical map, 3-folds of general type, Albanese map.

AMS subject classifications. 14J30, 14C20.

1. Introduction. We work over the complex number field $\mathbb{C}$.

The main purpose of this note is to study the geometry of Gorenstein minimal 3folds $X$ of general type. We improve the inequality $\chi\left(\omega_{X}\right) \leq 2 p_{g}(X)$ (see Proposition 2.1 for a precise statement), and we show how this leads to several applications which we explain below:

First, we improve the main theorem in [8]:

TheOREM 1.1. Let $X$ be a minimal projective Gorenstein 3-fold of general type. Assume that the canonical map $\Phi_{\left|K_{X}\right|}$ is of fiber type. Let $F$ be a smooth model of a generic irreducible component in the general fiber of $\Phi_{\left|K_{X}\right|}$. Then the invariants of $F$ are bounded from above as follows:

(1) if $F$ is a curve, then $g(F) \leq 487$;

(2) if $F$ is a surface, then $p_{g}(F) \leq 434$.

REMARK 1.2. 1) Theorem 1.1 was verified by the first author in [8] under the assumption that $p_{g}(X)$ is sufficiently large.

2) When $\Phi_{\left|K_{X}\right|}$ is generically finite, the generic degree is bounded from above by the second author in [11].

3) In the surface case, the corresponding boundedness theorem was proved by Beauville in [1].

4) The numerical bounds in the above theorem might be far from sharp.

Our second application is an inequality of Noether type between $c_{1}$ and $c_{2}$ which improves the main theorem of [17].

THEOREM 1.3. Let $X$ be a nonsingular projective minimal 3-fold of general type. Then the following inequality holds:

$$
K_{X}^{3} \geq \frac{8}{9} \chi\left(\omega_{X}\right)-\frac{10}{3}, \text { or equivalently }
$$

\footnotetext{
* Received October 21, 2005; accepted for publication February 24, 2006.

$\dagger$ Institute of Mathematics, School of Mathematical Sciences, Fudan University, Shanghai, 200433, People's Republic of China (mchen@fudan.edu.cn). The first author was supported both by Program for New Century Excellent Talents in University (Ministry of Education, PR China) and by the National Natural Science Foundation of China (no: 10131010).

${ }^{\ddagger}$ Department of Mathematics, University of Utah, 155 South 1400 East, Room 233, Salt Lake City, Utah 84112-0090, USA (hacon@math.utah.edu). The second author was partially supported by NSF research grant no: 0456363.
} 


$$
c_{1}^{3} \leq \frac{1}{27} c_{1} c_{2}+\frac{10}{3} .
$$

Chen is grateful to De-Qi Zhang for pointing out an inequality (see the proof of Lemma 2.1(3) in [19]) similar to the one in Proposition 2.1 and for an effective discussion.

2. Proof of Theorem 1.1. Throughout this note, a minimal 3-fold $X$ is one with nef canonical divisor $K_{X}$ and with only $\mathbb{Q}$-factorial terminal singularities.

2.1. Notations and the set up. Let $X$ be a minimal projective 3 -fold of general type. Since we are discussing the behavior of the canonical map, we may assume $p_{g}(X) \geq 2$. Denote by $\varphi_{1}$ the canonical map which is usually a rational map. Take the birational modification $\pi: X^{\prime} \longrightarrow X$, which exists by Hironaka's big theorem, such that

(i) $X^{\prime}$ is smooth;

(ii) the movable part of $\left|K_{X^{\prime}}\right|$ is base point free;

(iii) there exists a canonical divisor $K_{X}$ such that $\pi^{*}\left(K_{X}\right)$ has support with only normal crossings.

Denote by $h$ the composition $\varphi_{1} \circ \pi$. So $h: X^{\prime} \longrightarrow W^{\prime} \subseteq \mathbb{P}^{p_{g}(X)-1}$ is a morphism. Let $h: X^{\prime} \stackrel{f}{\longrightarrow} B \stackrel{s}{\longrightarrow} W^{\prime}$ be the Stein factorization of $h$. We can write

$$
K_{X^{\prime}}=\pi^{*}\left(K_{X}\right)+E=S+Z
$$

where $S$ is the movable part of $\left|K_{X^{\prime}}\right|, Z$ is the fixed part and $E$ is an effective $\mathbb{Q}$-divisor which is a sum of distinct exceptional divisors.

If $\operatorname{dim} \varphi_{1}(X)<3, f$ is a called an induced fibration of $\varphi_{1}$. If $\operatorname{dim} \varphi_{1}(X)=2$, a general fiber $F$ of $f$ is a smooth curve $C$ of genus $g:=g(C) \geq 2$. If $\operatorname{dim} \varphi_{1}(X)=1$, a general fiber $F$ of $f$ is a smooth projective surface of general type. Denote by $F_{0}$ the smooth minimal model of $F$ and by $\sigma: F \longrightarrow F_{0}$ the smooth blow down map. Denote by $b$ the genus of the base curve $B$.

Proposition 2.1. Let $V$ be a smooth projective 3-fold of general type with $p_{g}(V)>0$. Then $\chi\left(\omega_{V}\right) \leq p_{g}(V)$ unless a generic irreducible component in the general fiber of the Albanese morphism is a surface $V_{y}$ with $q\left(V_{y}\right)=0$, in which case one has the inequality

$$
\chi\left(\omega_{V}\right) \leq\left(1+\frac{1}{p_{g}\left(V_{y}\right)}\right) p_{g}(V) .
$$

Proof. Since $\chi\left(\omega_{V}\right)=p_{g}(V)+q(V)-h^{2}\left(\mathcal{O}_{V}\right)-1$, the result is clear if $q(V) \leq 1$. So assume that $q(V) \geq 2$. Let $a: V \rightarrow Y$ be the Stein factorization of the Albanese morphism $V \rightarrow A(V)$. By the proof of Theorem 1.1 in [11], one sees that we may assume that $\operatorname{dim} Y=1$ and hence $Y$ is a smooth curve. Recall also that by [11], $p_{g}(V) \geq \chi\left(a_{*} \omega_{V}\right)$. Let $y \in Y$ be a general point and $V_{y}$ the corresponding fiber. $V_{y}$ is a smooth surface of general type. If $q\left(V_{y}\right)>0$, then proceeding as in [11], one sees that $\chi\left(R^{1} a_{*} \omega_{V}\right)=\chi\left(R^{1} a_{*} \omega_{V / Y} \otimes \omega_{Y}\right)$. Since the genus of $Y$ is $q(V)$, and $\operatorname{deg} R^{1} a_{*} \omega_{V / Y} \geq 0$, one sees by an easy Riemann-Roch computation that

$$
\chi\left(R^{1} a_{*} \omega_{V}\right) \geq(q(V)-1) q\left(V_{y}\right) .
$$


Recall that $R^{2} a_{*} \omega_{V} \cong \omega_{Y}$ and so

$$
\chi\left(\omega_{V}\right)=\chi\left(a_{*} \omega_{V}\right)-\chi\left(R^{1} a_{*} \omega_{V}\right)+\chi\left(R^{2} a_{*} \omega_{V}\right) \leq \chi\left(a_{*} \omega_{V}\right) \leq p_{g}(V)
$$

whenever $q\left(V_{y}\right)>0$.

We may therefore assume that $q\left(V_{y}\right)=0$. Notice that by [14], the sheaf $R^{1} a_{*} \omega_{V}$ is torsion free. Since its rank is given by $h^{1}\left(\omega_{V_{y}}\right)=q\left(V_{y}\right)=0$, we have that $R^{1} a_{*} \omega_{V}=0$. Therefore, by a similar Riemann-Roch computation, one sees that $\chi\left(a_{*} \omega_{V}\right) \geq(q(V)-$ 1) $p_{g}\left(V_{y}\right)$ and so

$$
\chi\left(\omega_{V}\right)=\chi\left(a_{*} \omega_{V}\right)+q(V)-1 \leq \chi\left(a_{*} \omega_{V}\right)\left(1+\frac{1}{p_{g}\left(V_{y}\right)}\right) \leq p_{g}(V)\left(1+\frac{1}{p_{g}\left(V_{y}\right)}\right) .
$$

(

EXAMPLE 2.2. Let $S$ be a minimal surface of general type admitting a $\mathbb{Z}_{2}$ action such that $q(S)=0, p_{g}(S)=1$ and $p_{g}\left(S / \mathbb{Z}_{2}\right)=0$ (cf. (2.6) of [10]). Let $C$ be a curve admitting a fixed point free $\mathbb{Z}_{2}$ action and let $B=C / \mathbb{Z}_{2}$. Assume that the genus of $B$ is $b \geq 2$. Let $V=S \times C / \mathbb{Z}_{2}$ be the quotient by the induced diagonal action. Then $V$ is minimal, Gorenstein of general type such that $p_{g}(V)=b-1, q(V)=b$ and $h^{2}\left(\mathcal{O}_{V}\right)=0$. It follows that $\chi\left(\omega_{V}\right)=2 b-2=\left(1+1 / p_{g}\left(V_{y}\right)\right) p_{g}(V)$.

This example shows that the above proposition is close to being optimal.

Lemma 2.3. Let $X$ be a minimal 3-fold of general type. Suppose $\operatorname{dim} \varphi_{1}(X)=1$. Keep the same notations as in 2.1. Replace $\pi: X^{\prime} \longrightarrow X$, if necessary, by a further birational modification (we still denote it by $\pi$ ). Then

$$
\left.\pi^{*}\left(K_{X}\right)\right|_{F}-\frac{p_{g}(X)-1}{p_{g}(X)} \sigma^{*}\left(K_{F_{0}}\right)
$$

is pseudo-effective.

Proof. One has an induced fibration $f: X^{\prime} \longrightarrow B$.

Case 1. If $b>0$, we may replace $\pi$ by a new one as in the proof of Lemma 2.2 of [9] such that $\left.\pi^{*}\left(K_{X}\right)\right|_{F} \sim \sigma^{*}\left(K_{F_{0}}\right)$. In fact, since the fibers of $\pi$ are rationally connected $^{1}$ and $b>0$, it follows that $f: X^{\prime} \rightarrow B$ factors through a morphism $f_{1}: X \rightarrow B$. But since $X$ is minimal and terminal, it follows that a general fiber $X_{b}$ of $f_{1}$ is a smooth minimal surface of general type and hence it can be identified with $F_{0}$. It is now clear that $\left.\pi^{*}\left(K_{X}\right)\right|_{F} \sim \sigma^{*}\left(K_{F_{0}}\right)$.

Thus it suffices to consider the case $b=0$.

Case 2. If $p_{g}(X)=2$, the lemma was verified in section 4 (at page 526 and page $527)$ in [5]. If $p_{g}(X) \geq 3$, one may refer to Lemma 3.4 in [9].

Proposition 2.4. Let $X$ be a Gorenstein minimal projective 3-fold of general type. Let $d:=\operatorname{dim} \varphi_{1}(X)$. The following inequalities hold:

(1) If $d=2$, then $K_{X}^{3} \geq\left\ulcorner\frac{2}{3}(g(C)-1)\right\urcorner\left(p_{g}(X)-2\right)$.

(2) If $d=1$, then $K_{X}^{3} \geq\left(\frac{p_{g}(X)-1}{p_{g}(X)}\right)^{2} K_{F_{0}}^{2}\left(p_{g}(X)-1\right)$.

Proof. The inequality (1) is due to Theorem 4.1(ii) in [6].

\footnotetext{
${ }^{1}$ Shokurov ([18]) proved that if the pair $(X, \Delta)$ is klt and the MMP holds, then the fibres of the exceptional locus are always rationally chain connected. Furthermore, the second author and $\mathrm{M}^{\mathrm{C}}$ Kernan (see [12]) have recently extended Shokurov's result to any dimension and without assuming MMP.
} 
Suppose now that $d=1$. We may write

$$
\pi^{*}\left(K_{X}\right) \sim S+E_{\pi}
$$

where $S \equiv t F$ with $t \geq p_{g}(X)-1$ and $E_{\pi}$ is an effective divisor.

Thus we have

$$
\begin{aligned}
K_{X}^{3} & =\pi^{*}\left(K_{X}\right)^{3} \geq\left(\pi^{*}\left(K_{X}\right)^{2} \cdot F\right)\left(p_{g}(X)-1\right) \\
& \geq\left(\frac{p_{g}(X)-1}{p_{g}(X)}\right)^{2} \sigma^{*}\left(K_{F_{0}}\right)^{2}\left(p_{g}(X)-1\right)
\end{aligned}
$$

where Lemma 2.3 has been applied to derive the second inequality above.

2.2. Proof of Theorem 1.1. The Miyaoka-Yau inequality (cf. [15]) says

$$
K_{X}^{3} \leq 72 \chi\left(\omega_{X}\right)
$$

(**) Denote by $V$ a smooth model of $X$. Assume that a generic irreducible component in the general fiber of the Albanese morphism is a surface $V_{y}$ with $q\left(V_{y}\right)=0$ and $p_{g}\left(V_{y}\right)=1$. Because $p_{g}(V)=$ $p_{g}(X) \geq 2$, we see that the canonical map of $V$ maps $V_{y}$ to a point. This means $\operatorname{dim} \varphi_{1}(X)=1$, i.e. $\left|K_{X}\right|$ is composed with a pencil. Thus, one sees that in this special situation, the Stein factorization of the Albanese map is the fibration induced by $\varphi_{1}$. So $p_{g}(F)=$ $p_{g}\left(V_{y}\right)=1$.

(1) Assume $\operatorname{dim} \varphi_{1}(X)=2$. The above argument implies that $\chi\left(\omega_{X}\right) \leq \frac{3}{2} p_{g}(X)$ and so by proposition 2.4 and an easy computation, one sees that $g(C) \leq 487$. Furthermore $g(C) \leq 109$ whenever $p_{g}(X)$ is sufficiently big.

(2) Assume $\operatorname{dim} \varphi_{1}(X)=1$. When $b>0$, we have $p_{g}(F) \leq 38$ by (both 1.4 and Theorem 1.3 in) [8]. So we only need to study the case $b=0$.

Suppose $p_{g}(F) \geq 2$. Then one has $\chi\left(\omega_{X}\right) \leq \frac{3}{2} p_{g}(X)$ by argument $(* *)$ and Proposition 2.1. The Miyaoka-Yau inequality yields $K_{X}^{3} \leq 72 \chi\left(\omega_{X}\right) \leq 108 p_{g}(X)$. Again by Propositions 2.1 and 2.4, we have

$$
K_{F_{0}}^{2} \leq 108\left(\frac{p_{g}(X)}{p_{g}(X)-1}\right)^{3} \leq 864
$$

Also $K_{F_{0}}^{2} \leq 108$ whenever $p_{g}(X)$ is sufficiently big. Taking into account the Noether inequality $K_{F_{0}}^{2} \geq 2 p_{g}(F)-4$, we get $p_{g}(F) \leq 434$. This concludes the proof.

\section{A Noether type inequality between $c_{1}$ and $c_{2}$.}

3.1. A known inequality. Let $X$ be a nonsingular projective minimal 3 -fold of general type. We have a sharp inequality

$$
K_{X}^{3} \geq \frac{4}{3} p_{g}(X)-\frac{10}{3}
$$

which was first proved in [7] under the assumption $K_{X}$ being ample. The general case was recently proved in [2]. 


\subsection{Proof of Theorem 1.3.}

Proof. Note that since $K_{X}^{3}>0$ is an even integer, the Theorem clearly holds for $\chi\left(\omega_{X}\right) \leq 6$. Therefore, we may assume that $\chi\left(\omega_{X}\right)>0$.

Case 1. $p_{g}(X)>0$.

According to Proposition 2.1, we have an inequality $\chi\left(\omega_{X}\right) \leq \frac{3}{2} p_{g}(X)$ unless a generic irreducible component in the general fiber of the Albanese morphism is a surface $V_{y}$ with $q\left(V_{y}\right)=0$ and $p_{g}\left(V_{y}\right)=1$.

So in the general case by 3.1 one has the inequality

$$
K_{X}^{3} \geq \frac{8}{9} \chi\left(\omega_{X}\right)-\frac{10}{3}
$$

or equivalently,

$$
c_{1}^{3} \leq \frac{1}{27} c_{1} c_{2}+\frac{10}{3}
$$

In the exceptional case with $p_{g}(X)>1$, the argument (**) in 2.2 says that $\left|K_{X}\right|$ is composed with a pencil of surfaces and $\varphi_{1}$ generically factors through the Albanese map. Thus $X$ is canonically fibred by surfaces with $q\left(V_{y}\right)=0$ and $p_{g}\left(V_{y}\right)=1$. According to Theorem 4.1(iii) in [6], one has $K_{X}^{3} \geq 2 p_{g}(X)-4$. Since by Proposition $2.1 \chi\left(\omega_{X}\right) \leq 2 p_{g}(X)$, one has the stronger inequality $K_{X}^{3} \geq \chi\left(\omega_{X}\right)-4$.

In the exceptional case with $p_{g}(X)=1$, by Proposition 2.1, one has $\chi\left(\omega_{X}\right) \leq 2$ and so the inequality in Theorem 2.4 holds.

Case 2. $p_{g}(X)=0$.

We can not rely on 3.1 in this case. Since $\chi\left(\omega_{X}\right)>0$, one has $q(X)>1$. Thus we can study the Albanese map. Let $a: X \longrightarrow Y$ be the Stein factorization of the Albanese morphism. We claim that $\operatorname{dim}(Y)=1$. In fact, if $\operatorname{dim}(Y) \geq 2$, then the Proof of Theorem 1.1 of [11] shows $p_{g}(X) \geq \chi\left(a_{*} \omega_{X}\right) \geq \chi\left(\omega_{X}\right)>0$, a contradiction.

So we have a fibration $a: X \longrightarrow Y$ onto a smooth curve $Y$ with $g(Y)=q(X)>1$. Denote by $F$ a general fiber of $a$. If $p_{g}(F)>0$, then the Proof of Theorem 1.1 of [11] also shows $0=2 p_{g}(X) \geq \chi\left(\omega_{X}\right)>0$, which is also a contradiction. Thus one must have $p_{g}(F)=0$. Because $F$ is of general type, one has $q(F)=0$. Therefore, the sheaves $a_{*} \omega_{X}$ and $R^{1} a_{*} \omega_{X}$ have rank $h^{0}\left(\omega_{F}\right)=p_{g}(F)=0$ and $h^{1}\left(\omega_{F}\right)=q(F)=0$. Since, by [14], they are torsion free, it follows that they are both zero. So

$$
\chi\left(\omega_{X}\right)=\chi\left(R^{2} a_{*} \omega_{X}\right)=\chi\left(\omega_{Y}\right)=q(X)-1 .
$$

Still looking at the fibration $a: X \longrightarrow Y$, one sees that $a$ is relatively minimal since $X$ is minimal. Therefore $K_{X / Y}$ is nef by Theorem 1.4 of [16]. Thus one has $K_{X}^{3} \geq(2 q(X)-2) K_{F}^{2} \geq 2 \chi\left(\omega_{X}\right)$, which is stronger than the required inequality.

4. Examples. In Example 2(e) of [4], one may find a smooth projective 3-fold of general type which is composed with a pencil of surfaces of $p_{g}(F)=5$, the biggest value among known examples. Here we present another example which is composed with curves of genus $g=5$.

EXAMPLE 4.1. We follow the Example in $\S 4$ of [3]. We consider bi-double covers $f_{i}: C_{i} \rightarrow E_{i}$ of curves where, $g\left(E_{i}\right)=0,0,2$. We assume that

$$
\left(d_{i}\right)_{*} \mathcal{O}_{C_{i}}=\mathcal{O}_{E_{i}} \oplus L_{i}^{\vee} \oplus P_{i}^{\vee} \oplus L_{i}^{\vee} \otimes P_{i}^{\vee}
$$


where for we have $\operatorname{deg}\left(L_{1}\right)=d_{1}, \operatorname{deg}\left(L_{2}\right)=d_{2}, \operatorname{deg}\left(P_{1}\right)=\operatorname{deg}\left(P_{2}\right)=1$ and $L_{3}, P_{3}$ are distinct 2-torsion elements in $\mathrm{Pic}^{0}\left(E_{3}\right)$. In particular $g\left(C_{i}\right)=2 d_{i}-1$ for $i \in\{1,2\}$ and $f_{3}$ is étale. It follows that

$$
\delta: D_{1} \times D_{2} \times D_{3} \rightarrow E_{1} \times E_{2} \times E_{3}
$$

is a $\mathbb{Z}_{2}^{6}$ cover. We denote by $l_{i}, p_{i}, l_{i} p_{i}$ the elements of $\mathbb{Z}_{2}^{2}$ whose eigensheaves with eigenvalues 1 are $L_{i}^{\vee}, P_{i}^{\vee}$ and $\left(L_{i} \otimes P_{i}\right)^{\vee}$. Let $X:=D_{1} \times D_{2} \times D_{3} / G$ where $G \cong \mathbb{Z}_{2}^{4}$ is the group generated by

$$
\left\{\left(1, p_{2}, l_{3}\right),\left(p_{1}, l_{2}, 1\right),\left(l_{1}, 1, p_{3}\right),\left(p_{1}, p_{2}, p_{3}\right)\right\} .
$$

Then one sees that $X$ is Gorenstein and for the induced morphism $f: X \rightarrow E_{1} \times$ $E_{2} \times E_{3}$, one has

$$
\begin{gathered}
f_{*} \mathcal{O}_{X}=\left(\delta_{*} \mathcal{O}_{D_{1} \times D_{2} \times D_{3}}\right)^{G} \cong \mathcal{O}_{E_{1}} \times \mathcal{O}_{E_{2}} \times \mathcal{O}_{E_{3}} \oplus \\
\left(L_{1}^{\vee} \otimes L_{2}^{\vee} \otimes P_{2}^{\vee} \otimes P_{3}^{\vee}\right) \oplus\left(P_{1}^{\vee} \otimes L_{2}^{\vee} \otimes L_{3}^{\vee} \otimes P_{3}^{\vee}\right) \oplus\left(L_{1}^{\vee} \otimes P_{1}^{\vee} \otimes P_{2}^{\vee} \otimes L_{3}^{\vee}\right) .
\end{gathered}
$$

Since $f_{*} \omega_{X}=\omega_{E_{1} \times E_{2} \times E_{3}} \otimes f_{*} \mathcal{O}_{X}$, it follows easily that

$$
H^{0}\left(\omega_{X}\right) \cong H^{0}\left(\omega_{E_{1}} \otimes L_{1}\right) \otimes H^{0}\left(\omega_{E_{2}} \otimes L_{2} \otimes P_{2}\right) \otimes H^{0}\left(\omega_{E_{3}} \otimes P_{3}\right) .
$$

In particular $p_{g}(X)=\left(d_{1}-1\right)\left(d_{2}-1\right)$ and $\varphi_{1}$ factors through the map $X \rightarrow C_{1} / \mathbb{Z}_{2} \times$ $C_{2} / \mathbb{Z}_{2}$. The fibers of $\varphi_{1}$ are then isomorphic to $C_{3}$ and hence have genus 5 .

Question 4.2. A very natural open problem is to find sharp upper bounds of the invariants of $F$ in Theorem 1.1. It is very interesting to find a new example $X$ which is a Gorenstein minimal 3-fold of general type such that $\Phi_{\left|K_{X}\right|}$ is of fiber type and that the generic irreducible component in a general fiber has larger birational invariants.

We remark that the above question is still open in the surface case. So Question 4.2 is probably quite difficult. A first step should be to construct new examples with bigger fiber invariants.

\section{REFERENCES}

[1] A. Beauville, L'application canonique pour les surfaces de type général, Invent. Math., 55 (1979), pp. 121-140.

[2] F. Catanese, M. Chen, D.-Q. Zhang, The Noether inequality for smooth minimal 3-folds, Math. Res. Lett., 13 (2006), pp. 653-666.

[3] J. A. Chen, C. D. Hacon, On the irregularity of the image of the Iitaka fibration, Comm. in Algebra, 32:1 (2004), pp. 203-215.

[4] M. Chen, Z. J. Chen, Irregularity of canonical pencils for a threefold of general type, Math. Proc. Camb. Phil. Soc., 125 (1999), pp. 83-87.

[5] M. Chen, Canonical stability of 3-folds of general type with $p_{g} \geq 3$, Internat. J. Math., 14 (2003), pp. 515-528.

[6] M. Chen, Inequalities of Noether type for 3-folds of general type, J. Math. Soc. Japan, 56 (2004), pp. 1131-1155.

[7] M. Chen, Minimal 3-folds of small slope and the Noether inequality for canonically polarized 3-folds, Math. Res. Lett., 11 (2004), pp. 833-852.

[8] M. Chen, Weak boundedness theorems for canonically fibered Gorenstein minimal 3-folds, Proc. Amer. Math. Soc., 133:5 (2005), pp. 1291-1298. 
[9] M. Chen, A sharp lower bound for the canonical volume of 3-folds of general type, Math. Ann. (to appear), math.AG/0407397.

[10] C. D. Hacon, Effective criteria for birational morphisms, Jour. London Math. Soc., 67:2 April (2003), pp. 337-348.

[11] C. D. HAcon, On the degree of the canonical maps of 3-folds, Proc. Japan Acad. Ser. A Math. Sci., $80: 8$ (2004), pp. 166-167.

[12] C. D. HACON, J. M ${ }^{c}$ KeRnAN, Shokurov's rational connectedness conjecture, Invent. Math., 166 (2006), pp. 1-25.

[13] Y. Kawamata, K. Matsuda, K. Matsuki, Introduction to the minimal model problem, Adv. Stud. Pure Math., 10 (1987), pp. 283-360.

[14] J. Kollár, Higher direct images of dualizing sheaves I, Ann. Math., 123 (1986), pp. 11-42.

[15] Y. MiYaokA, The pseudo-effectivity of $3 c_{2}-c_{1}^{2}$ for varieties with numerically effective canonical classes, Algebraic Geometry, Sendai, 1985. Adv. Stud. Pure Math., 10 (1987), pp. 449-476.

[16] K. Онnо, Some inequalities for minimal fibrations of surfaces of general type over curves, J. Math. Soc. Japan, 44 (1992), pp. 643-666.

[17] D.-K. Shin, Some inequalities between $K_{X}^{3}$ and $\chi\left(\mathcal{O}_{X}\right)$ for a threefold $X$ of general type, Math. Z., 225:1 (1997), pp. 133-138.

[18] V. V. Shokurov, On rational connectedness, Math. Notes, 68 (2000), pp. 652-660.

[19] D.-Q. Zhang, Small bound for birational automorphism groups of algebraic varieties (with an appendix by Yujiro Kawamata), Math. Annalen (to appear), math.AG/0609083. 
